\title{
PERFORMANCE AVAILABILITY ASSESSMENT OF COMBINED MULTI POWER SOURCE TRACTION DRIVE CONSIDERING REAL OPERATIONAL CONDITIONS
}

\author{
Ilia Frenkel ${ }^{1}$, Igor Bolvashenkov ${ }^{2}$, Hans-Georg Herzog ${ }^{2}$, Lev Khvatskin ${ }^{1}$ \\ ${ }^{1}$ Center for Reliability and Risk Management \\ Industrial Engineering and Management Department \\ SCE - Shamoon College of Engineering \\ Beer-Sheva, Israel \\ ${ }^{2}$ Institute of Energy Conversion Technology \\ Technical University of Munich \\ Munich, Germany
}

\begin{abstract}
The present paper deals with the vehicle's traction electric drive, consisting of several various electric power sources. One of the main requirements for such systems are the safety and sustainable operations, achieved largely the implementation of an uninterrupted supply of the vehicle's propulsion system with an electric power.

One way of realization of the required level of operational sustainability is usage of Multi Power Source Traction Drive. This type of vehicle's propulsion systems is widely applied on the ships, trains, planes, heavy trucks. The most important impact on the sustainability of functioning of the ship's propulsion system have operational conditions, especially for the cargo ship, operating in Arctic region.

This paper presents the application of the $L_{Z}$-transform method to assessing the important parameter of the vehicle's operational sustainability - availability of the multi-state (MSS) Multi Power Source Traction Drive, which includes Diesel-Generators and Gas-Turbine-Generators considering the impact of the real operating conditions.

Straightforward Markov method applied to solve this problem will require building of a system's model with numerous numbers of states and solve a corresponding system of multiple differential equations. $L_{Z}$-transform method, drastically simplified the solution.
\end{abstract}

Keywords: Multi Power Source Traction Drive, Lz-transform method, Multi-state systems, Assessing Operational Sustainability, Arctic cargo ship, Electric Propulsion System

\section{Introduction}

At the present time according to the program of the electrification of various types of vehicles based on the electric energy, generated by renewable sources, the usage of different types of hybrid (dieselelectric, gasoline-electric etc.) and electrical traction systems for the various types of vehicles have now become very relevant (Bolvashenkov and Herzog, 2016; Bolvashenkov et al., 2014). On the other hand, the constant growth of complexity of modern engineering systems does more and more complicated the task of achieving the required level of its safety and sustainable operations. The implementation of the specified requirements closely related to the assessment of sustainable operation indicators of the system. Indicators for sustainable operation are reliability and safety. Quantitative indicators of reliability are failure free operation probability, availability, repairability and durability. Quantitative indicators of safety are fault tolerance, survivability, resiliency and stability.

The greatest interest has a quantitative assessment of performance availability indices depending on the condition of the vehicle's operation, because they allow systematically evaluate such comprehensive indicators as the safety and sustainability.

Due to the fact that the vehicle's traction drives are the safety-critical systems, it is particularly important to assess correctly and accurately the required sustainability indices (safety and reliability) for the real conditions of operation. The values of these indices for the each equipment's elements should satisfy the requirements of the project.

In recent years, a specific approach called the universal generating function (UGF) technique has been widely applied to MSS reliability analysis (Lisnianski et al., 2010; Ushakov, 1986). However, the main restriction of this powerful technique is that it may only be applied to random variables and therefore, concerning MSS reliability, it operates only with steady-states performance distributions. In order to extend the UGF technique application to dynamic MSS reliability analysis a special transform, called $L_{\mathrm{Z}}$-transform, 
was introduced (Lisnianski, 2012) for discrete-state continuous-time Markov processes, where one can find its detailed description and corresponding mathematical proofs.

In the present paper, the $L_{Z}$-transform is applied to a real MSS Multi Power Source Traction Drive and its availability is analyzed. It is shown that $L_{Z}$-transform application drastically simplifies the availability computation for such a system compared with the straightforward Markov method.

\section{Brief Description of the $L_{z}$-transform Method}

We consider a discrete-state continuous-time (DSCT) Markov process $X(t) \in\left\{x_{1}, \ldots, x_{K}\right\}$, which has $K$ possible states $i,(i=1, \ldots, K)$ where the performance level associated with any state $i$ is $x_{i}$. This Markov process is completely defined by the set of possible states $\mathbf{x}=\left\{x_{1}, x_{2}, \ldots, x_{K}\right\}$, the transition intensities matrix depending on time $A=\left(a_{i j}(t)\right), i, j=1,2, \ldots, K$ and by the initial states probability distribution given by $\mathbf{p}_{0}=\left[p_{10}=\operatorname{Pr}\left\{X(0)=x_{1}\right\}, \ldots, p_{K 0}=\operatorname{Pr}\left\{X(0)=x_{K}\right\}\right]$.

According to (Lisnianski, 2012), the $L_{Z}$-transform of a DSCT Markov process $X(t)$ is defined by the following expression

$$
L_{z}\{X(t)\}=\sum_{i=1}^{K} p_{i}(t) z^{g_{i}}
$$

where $p_{i}(t)$ is the probability that the process is in state $i$ at time instant $t \geq 0$ for a given initial states probability distribution $\mathbf{p}_{0}, g_{i}$ is the performance level of state $i$, and $z$ is a complex variable.

In general, any element $j$ in MSS can have $k_{j}$ different states corresponding to different performance, represented by the set $\mathbf{g}_{j}=\left\{g_{j 1}, \ldots, g_{j k_{j}}\right\}$, where $g_{j i}$ is the performance rate of element $j$ in the state $i$, $i \in\left\{1,2, \ldots, k_{j}\right\}$, and $j \in\{1, \ldots n\}$, where $n$ is the number of elements in the MSS.

According to $L_{Z}$-transform method at first stage, a Markov model of stochastic process should be built for each multi-state element in MSS. Based on this model, state probabilities $p_{j i}(t)=\operatorname{Pr}\left\{G_{j}(t)=g_{j i}\right\}$, $i \in\left\{1, \ldots, k_{j}\right\}$ for every MSS's element can be obtained as a solution of the corresponding system of differential equations under the given initial conditions. These probabilities define output stochastic process $G_{j}(t)$ for each element $j$ in the MSS. Then, individual $L_{Z}$-transform for each element $j$ should be found

$L_{z}\left\{G_{j}(t)\right\}=\sum_{i=1}^{k_{j}} p_{j i}(t) z^{g_{j i}}, j=1, \ldots, n$.

At the next stage based on previously determined $L_{Z}$-transform for each element $j$ and system structure function $f$, given by $G(t)=f\left(G_{1}(t), \ldots, G_{n}(t)\right), L_{Z}$-transform of the output stochastic process for the entire MSS should be defined. Using Ushakov's operator $\Omega_{f}$ (Ushakov, 1986) over all $L_{Z}$-transforms of individual elements one can obtain the resulting $L_{Z}$-transform $L_{z}\{G(t)\}$ associated with output performance stochastic process $G(t)$ of the entire MSS:

$L_{z}\{G(t)\}=\Omega_{f}\left\{L_{z}\left[G_{1}(t)\right], \ldots, L_{z}\left[G_{n}(t)\right]\right\}$. MSS:

The resulting $L_{Z}$ transform is associated with the output performance stochastic process for the entire $L_{z}\{G(t)\}=\sum_{k=1}^{K} p_{k}(t) z^{g_{k}}$.

MSS mean instantaneous availability for constant demand level $w$ can be easily derived from the resulting $L_{\mathrm{Z}}$-transform in the following form:

$$
A(t)=\sum_{g_{k} \geq w} p_{k}(t)
$$


In other words, in order to find MSS's mean instantaneous availability one should summarize all probabilities in $L_{z}$-transform from terms where powers of $z$ are greater to demand level.

\section{Multi-state Model of the Multi Power Source Traction Drive with Gas Turbine}

\section{A. System Description}

We consider the combined Gas-Turbine- and Diesel-electric power drive using in arctic cargo ships and icebreakers. Structure of ship's traction drive is shown in Fig. 1. The system consists of Gas- TurbineGenerator and Diesel-Generators subsystem, Main Switchboard, Electric Energy Converter and Electric Motor Subsystem.

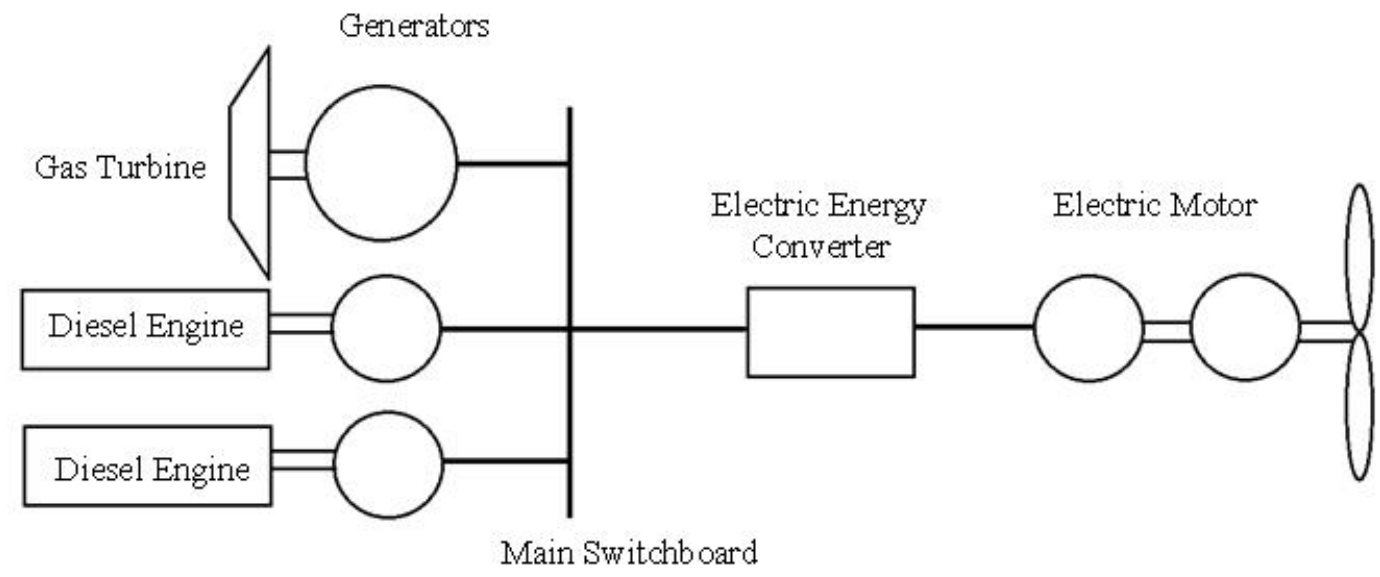

Figure 1. Structure of the ship's gas-turbine- and diesel-electric traction drive

Currently, the use of the Gas-Turbine-Generators as sources of electric energy onboard the vehicle is regarded as a very promising and competitive in comparison with conventional ship's Diesel-Generators.

The main advantages of the gas turbine considering (Bolvashenkov et al., 2014) are the follows:

- $\quad$ lower operational costs;

- large emissions reduction;

- lower vibrations and noise level;

- $\quad$ significant gain of weight and volume, especially for the ships with Azipod system.

Researches show that prime power generation on board hybrid electric ships presents several options that affect fuel consumption, power density, operational effectiveness, and survivability. It is found that direct coupling of gas turbines to permanent magnet generators reduces system mass and volume significantly as compared to conventional electric power generation systems installed today on the ships.

In the presented paper the authors have attempted to quantify the above advantages of the Gas Turbine in combined ship's traction drive in the real operational conditions on the basis of the performance availability calculation.

The nominal performance of the whole ship's propulsion system is $5500 \mathrm{KW}$.

Performance of Gas-Turbine-Generator is $2750 \mathrm{KW}$. Performance of each Diesel-Generator is 1375 KW. Therefore, connecting Gas-Turbine-Generator and two Diesel-Generators in parallel results in nominal performance, which is required for the functioning of the system.

Main Switchboard device and Electric Energy Converter have nominal performance.

Performance of each Electric Motor is $2750 \mathrm{KW}$. Therefore, connecting Electric Motors in parallel results in nominal performance, which is required for the functioning of the system.

In the ship's Gas-Turbine and diesel-electric power drives with a fix pitch propeller, the dimensioning of the electric machines has to be calculated accurately in order to estimate the available sufficient propulsion power, which directly determines by the required value of operational power and needed additional power in case of heavy weather or ice conditions in the area of navigation.

Possible structures of the arctic ship's propulsion system with a different number of generators and main traction motors are determined by operating conditions of the arctic ship and the ice and temperature conditions, look Fig. 2. 


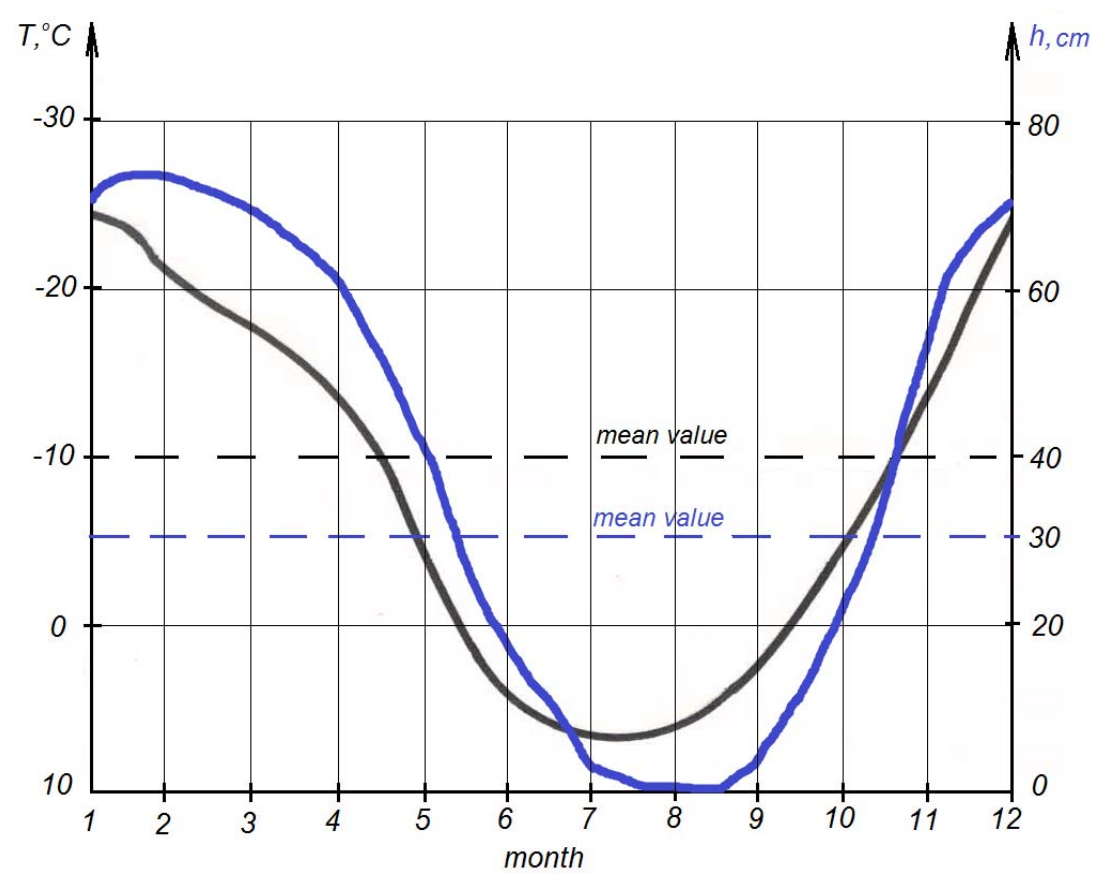

Figure 2. Ice and temperature conditions of Arctic navigation

According to collected statistical data during the long-term operations of the ships in ice conditions of the Arctic, operational modes of arctic cargo ships can be represented as follows:

- Navigation with ice-breaker in heavy ice and navigation without icebreaker in solid ice needs $100 \%$ of generated power;

- Navigation in the open water, depended on required velocity, needs the greater part of the operational time $50 \%$ of the generated power.

This situation is typical for the arctic cargo ships. Usually, for such systems, demand is $100 \%$ and $50 \%$ of nominal power performance.

The state-transition diagram of the Gas-Turbine and Diesel-electric power drive is presented in the Fig. 3.

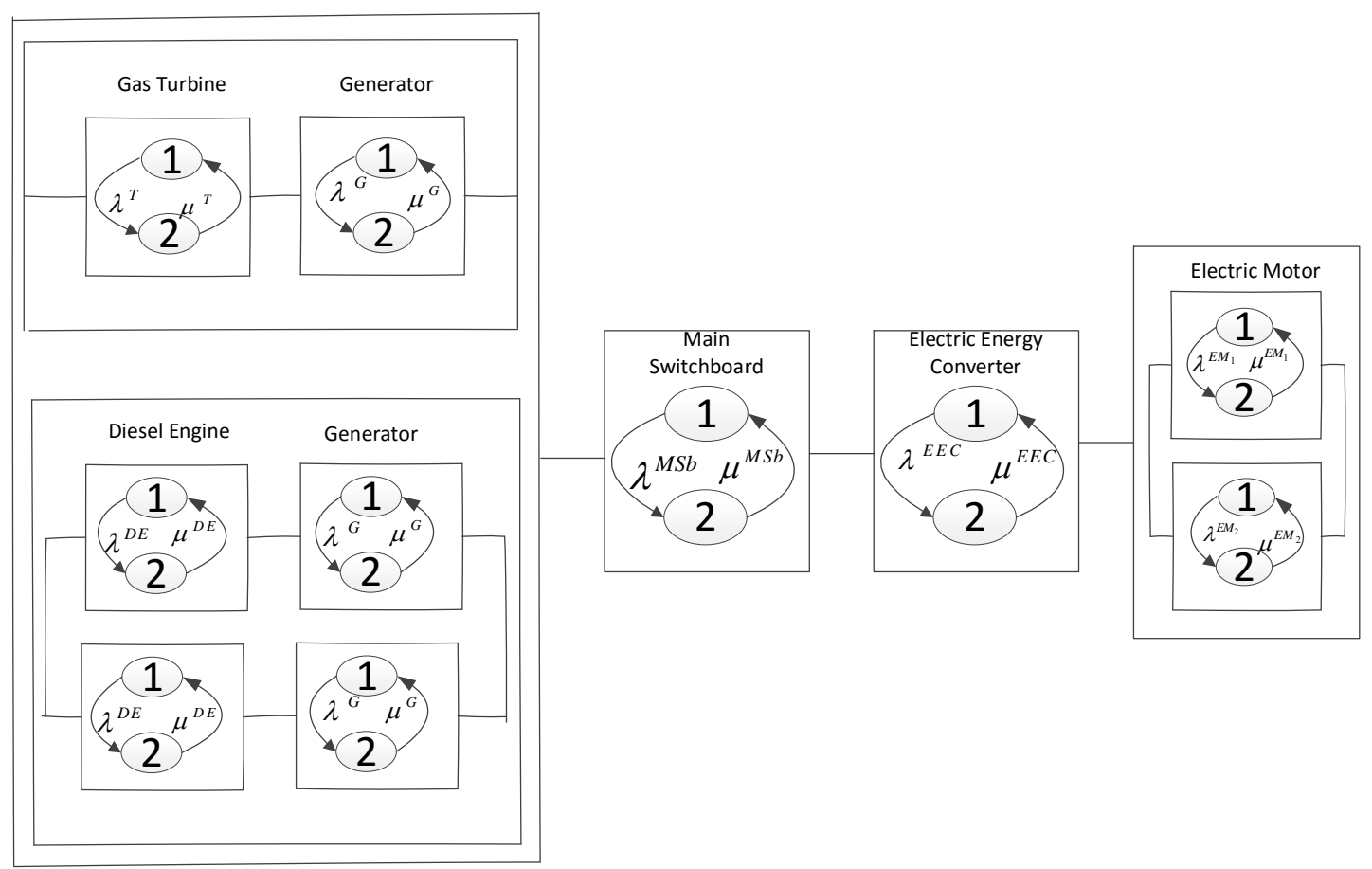

Figure 3. Structure and state-transition diagram of the Gas-Turbine and Diesel-electric power drive 


\section{B. Gas-Turbine-Diesel-Generator's sub-system}

Gas-Turbine-Diesel-Generator's sub-system consists of 3 connected in parallel pairs: Gas-TurbineEngine and Generator and 2 pairs of Diesel-Engine and Generator. Using the state-transitions diagram (Fig. 3) in accordance with the Markov method, we build the following systems of differential equations:

- for Gas-Turbine-Engine and Generator:

$$
\left\{\begin{array}{l}
\frac{d p_{1}^{G T E}(t)}{d t}=-\lambda^{G T E} p_{1}^{G T E}(t)+\mu^{G T E} p_{2}^{G T E}(t), \\
\frac{d p_{2}^{G T E}(t)}{d t}=\lambda^{G T E} p_{1}^{G T E}(t)-\mu^{G T E} p_{2}^{G T E}(t) .
\end{array}\right.
$$

Initial conditions are: $p_{1}^{G T E}(0)=1 ; p_{2}^{G T E}(0)=0$.

$$
\left\{\begin{array}{l}
\frac{d p_{1}^{G T G}(t)}{d t}=-\lambda^{G T G} p_{1}^{G T G}(t)+\mu^{G T G} p_{2}^{G T G}(t), \\
\frac{d p_{2}^{G T G}(t)}{d t}=\lambda^{G T G} p_{1}^{G T G}(t)-\mu^{G T G} p_{2}^{G T G}(t) .
\end{array}\right.
$$

Initial conditions are: $p_{1}^{G T G}(0)=1 ; p_{2}^{G T G}(0)=0$.

- for Diesel-Engine and Generator $(i=1,2)$ :

$$
\left\{\begin{array}{l}
\frac{d p_{i 1}^{D E}(t)}{d t}=-\lambda^{D E} p_{i 1}^{D E}(t)+\mu^{D E} p_{i 2}^{D E}(t), \\
\frac{d p_{i 2}^{D E}(t)}{d t}=\lambda^{D E} p_{i 1}^{D E}(t)-\mu^{D E} p_{i 2}^{D E}(t) .
\end{array}\right.
$$

Initial conditions are: $p_{i 1}^{D G}(0)=1 ; p_{i 2}^{D G}(0)=0$.

$$
\left\{\begin{array}{l}
\frac{d p_{i 1}^{G}(t)}{d t}=-\lambda^{G} p_{i 1}^{G}(t)+\mu^{G} p_{i 2}^{G}(t), \\
\frac{d p_{i 2}^{G}(t)}{d t}=\lambda^{G} p_{i 1}^{G}(t)-\mu^{G} p_{i 2}^{G}(t) .
\end{array}\right.
$$

Initial conditions are: $p_{i 1}^{G}(0)=1 ; p_{i 2}^{G}(0)=0$.

A numerical solution for probabilities $p_{1}^{G T E}(t), p_{2}^{G T E}(t), p_{1}^{G T G}(t), p_{2}^{G T G}(t), p_{i 1}^{D E}(t), p_{i 2}^{D E}(t)$ and $p_{i 1}^{G}(t), \quad p_{i 2}^{G}(t) \quad(i=1,2)$ can be obtained for each of these systems of differential equations using MATLAB ${ }^{\circledR}$. Therefore, for Gas-Turbine-Engine and Generator and each Diesel Engine and Generator we can obtain the following output performance stochastic processes:

$$
\begin{array}{ll} 
\begin{cases}\mathbf{g}^{G T E}=\left\{g_{1}^{G T E}, g_{2}^{G T E}\right\}=\{2750,0\}, & \mathbf{p}^{G T E}(t)=\left\{p_{1}^{G T E}(t), p_{2}^{G T E}(t)\right\} .\end{cases} & \left\{\begin{array}{l}
\mathbf{g}^{G T G}=\left\{g_{1}^{G T G}, g_{2}^{G T G}\right\}=\{2750,0\}, \\
\mathbf{p}^{G T G}(t)=\left\{p_{1}^{G T G}(t), p_{2}^{G T G}(t)\right\} .
\end{array}\right. \\
\left\{\begin{array}{l}
\mathbf{g}_{i}^{D E}=\left\{g_{i 1}^{D E}, g_{i 2}^{D E}\right\}=\{1375,0\}, \\
\mathbf{p}_{1}^{D E}(t)=\left\{p_{i 1}^{D E}(t), p_{i 2}^{D E}(t)\right\} .
\end{array}\right. & \left\{\begin{array}{l}
\mathbf{g}_{i}^{G}=\left\{g_{i 1}^{G}, g_{i 2}^{G}\right\}=\{1375,0\}, \\
\mathbf{p}_{1}^{G}(t)=\left\{p_{i 1}^{G}(t), p_{i 2}^{G}(t)\right\} .
\end{array}\right.
\end{array}
$$

Having the sets $\mathbf{p}^{G T E}(t), \mathbf{g}^{G T E}, \mathbf{p}^{G T G}(t), \mathbf{g}^{G T G}, \mathbf{g}_{i}^{D E}, \mathbf{p}_{i}^{D E}(t), \mathbf{g}_{i}^{G}, \mathbf{p}_{i}^{G}(t), i=1,2,3,4$ one can define for Gas-Turbine-Engine and Generator and each Diesel Engine and Generator $L_{z}$-transforms, associated with their output performance stochastic processes:

$$
\begin{array}{rrr}
L_{z}\left\{G^{G T E}(t)\right\}=p_{1}^{G T E}(t) z^{g_{1}^{G T E}}+p_{2}^{G T E}(t) z^{g_{2}^{G T E}} & L_{z}\left\{G^{G T G}(t)\right\}=p_{1}^{G T G}(t) z^{g_{1}^{G T G}}+p_{2}^{G T G}(t) z^{g_{2}^{G T G}} \\
=p_{1}^{G T E}(t) z^{2750}+p_{2}^{G T E}(t) z^{0} . & =p_{1}^{G T G}(t) z^{2750}+p_{2}^{G T G}(t) z^{0} . \\
L_{z}\left\{g_{i}^{D E}(t)\right\}=p_{i 1}^{D E}(t) z^{g_{i 1}^{D E}}+p_{i 2}^{D E}(t) z^{g_{i 2}^{D E}} & L_{z}\left\{g_{i}^{G}(t)\right\}=p_{i 1}^{G}(t) z^{g_{i 1}^{G}}+p_{i 2}^{D}(t) z^{g_{i 2}^{G}} \\
=p_{i 1}^{D E}(t) z^{1375}+p_{i 2}^{D E}(t) z^{0} . & =p_{i 1}^{G}(t) z^{1375}+p_{i 2}^{G}(t) z^{0} .
\end{array}
$$

Using the composition operator $\Omega_{\text {fer }}$ for Gas-Turbine-Engine and Generator, connected in series, one can obtain the $L_{Z}$-transform $L_{z}\left\{G^{\text {GTEG }}(t)\right\}$, where the powers of $z$ are found as minim of powers of corresponding terms: 


$$
\begin{aligned}
L_{z}\left\{G_{i}^{G T E G}(t)\right\} & =\Omega_{f_{\text {ser }}}\left(L_{z}\left\{G^{G T E}(t)\right\}, L_{z}\left\{G^{G T G}(t)\right\}\right) \\
& =\Omega_{f_{\text {ser }}}\left(p_{1}^{G T E}(t) z^{2750}+p_{2}^{G T E}(t) z^{0}, p_{1}^{G T G}(t) z^{2750}+p_{2}^{G T G}(t) z^{0}\right) \\
& =p_{1}^{G T E}(t) p_{1}^{G T G}(t) z^{2750}+\left(p_{1}^{G T E}(t) p_{2}^{G T G}(t)+p_{2}^{G T E}(t) p_{1}^{G T G}(t)+p_{2}^{G T E}(t) p_{2}^{G T G}(t)\right) z^{0} \\
& =p_{1}^{G T E}(t) p_{1}^{G T G}(t) z^{2750}+\left(p_{1}^{G T E}(t) p_{2}^{G T G}(t)+p_{2}^{G T E}(t)\right) z^{0}
\end{aligned}
$$

Using the following notations

$$
\begin{aligned}
& p_{1}^{G T E G}(t)=p_{1}^{G T E}(t) p_{1}^{G T G}(t) ; \\
& p_{2}^{G T E G}(t)=p_{1}^{G T E}(t) p_{2}^{G T G}(t)+p_{2}^{G T E}(t) ;
\end{aligned}
$$

one can obtain the resulting $L_{Z}$-transform for the Diesel-Generator's sub-system in the following form

$$
L_{z}\left\{G^{G T E G}(t)\right\}=p_{1}^{G T E G}(t) z^{2750}+p_{2}^{G T E G}(t) z^{0} .
$$

Using the composition operator $\Omega_{\text {fser }}$ for Diesel Engine and Generator, connected in series, one can obtain the $L_{Z}$-transform $L_{z}\left\{G^{D G}(t)\right\}$ for the Diesel-Generator's sub-system, where the powers of $z$ are found as minim of powers of corresponding terms:

$$
\begin{aligned}
L_{z}\left\{G_{i}^{D G}(t)\right\} & =\Omega_{f_{s e r}}\left(L_{z}\left\{g_{i}^{D E}(t)\right\}, L_{z}\left\{g_{i}^{G}(t)\right\}\right)= \\
& =\Omega_{f_{s e r}}\left(p_{i 1}^{D E}(t) z^{1375}+p_{i 2}^{D E}(t) z^{0}, p_{i 1}^{G}(t) z^{1375}+p_{i 2}^{G}(t) z^{0}\right) \\
& =p_{i 1}^{D E}(t) p_{i 1}^{G}(t) z^{1375}+\left(p_{i 1}^{D E}(t) p_{i 2}^{G}(t)+p_{i 2}^{D E}(t) p_{i 1}^{G}(t)+p_{i 2}^{D E}(t) p_{i 2}^{G}(t)\right) z^{0} \\
& =p_{i 1}^{D E}(t) p_{i 1}^{G}(t) z^{1375}+\left(p_{i 1}^{D E}(t) p_{i 2}^{G}(t)+p_{i 2}^{D E}(t)\right) z^{0} .
\end{aligned}
$$

Using the following notations

$$
\begin{aligned}
& p_{i 1}^{D G}(t)=p_{i 1}^{D E}(t) p_{i 1}^{G}(t) ; \\
& p_{i 2}^{D G}(t)=p_{i 1}^{D E}(t) p_{i 2}^{G}(t)+p_{i 2}^{D E}(t) ;
\end{aligned}
$$

one can obtain the resulting $L_{Z}$-transform for the Diesel-Generator's sub-system in the following form $L_{z}\left\{G_{i}^{D G}(t)\right\}=p_{i 1}^{D G}(t) z^{1375}+p_{i 2}^{D G}(t) z^{0}, i=1,2$.

Using the composition operator $\Omega_{\text {fpar }}$ for connected in parallel Gas-Turbine-Engine and Generator and two Diesel-Generators, one can obtain the $L_{Z}$-transform $L_{z}\left\{G^{G T D G}(t)\right\}$ for the Gas-Turbine-DieselGenerator's sub-system, where the powers of $z$ are found as sum of powers of corresponding terms:

$$
\begin{aligned}
L_{z}\left\{G^{G T D G}(t)\right\}= & \Omega_{f_{\text {par }}}\left(L_{z}\left\{G^{G T E G}(t)\right\}, L_{z}\left\{G_{1}^{D G}(t)\right\}, L_{z}\left\{G_{2}^{D G}(t)\right\}\right) \\
& =\Omega_{f_{\text {par }}}\left(p_{1}^{G T E G}(t) z^{2750}+p_{2}^{G T E G}(t) z^{0}, p_{11}^{D G}(t) z^{1375}+p_{12}^{D G}(t) z^{0}, p_{21}^{D G}(t) z^{1375}+p_{22}^{D G}(t) z^{0}\right) \\
& =p_{1}^{G T E G}(t) p_{11}^{D G}(t) p_{21}^{D G}(t) z^{5500} \\
& +p_{1}^{G T E G}(t)\left(p_{11}^{D G}(t) p_{22}^{D G}(t)+p_{12}^{D G}(t) p_{21}^{D G}(t)\right) z^{4175} \\
& +\left(p_{1}^{G T E G}(t) p_{12}^{D G}(t) p_{22}^{D G}(t)+p_{2}^{G T E G}(t) p_{11}^{D G}(t) p_{21}^{D G}(t)\right) z^{2750} \\
& +p_{2}^{G T E G}(t)\left(p_{11}^{D G}(t) p_{22}^{D G}(t)+p_{12}^{D G}(t) p_{21}^{D G}(t)\right) z^{1375} \\
& +p_{2}^{G T E G}(t) p_{12}^{D G}(t) p_{22}^{D G}(t) z^{0} .
\end{aligned}
$$


Using the following notations

$$
\begin{aligned}
& P_{1}^{G T D G}(t)=p_{1}^{G T E G}(t) p_{11}^{D G}(t) p_{21}^{D G}(t) ; \\
& P_{2}^{G T D G}(t)=p_{1}^{G T E G}(t)\left(p_{11}^{D G}(t) p_{22}^{D G}(t)+p_{12}^{D G}(t) p_{21}^{D G}(t)\right) ; \\
& P_{3}^{G T D G}(t)=p_{1}^{G T E G}(t) p_{12}^{D G}(t) p_{22}^{D G}(t)+p_{2}^{G T E G}(t) p_{11}^{D G}(t) p_{21}^{D G}(t) ; \\
& P_{4}^{G T D G}(t)=p_{2}^{G T E G}(t)\left(p_{11}^{D G}(t) p_{22}^{D G}(t)+p_{12}^{D G}(t) p_{21}^{D G}(t)\right) ; \\
& P_{5}^{G T D G}(t)=p_{2}^{G T E G}(t) p_{12}^{D G}(t) p_{22}^{D G}(t),
\end{aligned}
$$

one can obtain the resulting $L_{Z}$-transform for the Gas-Turbine-Diesel-Generator's sub-system in the following form

$$
L_{z}\left\{G^{G T D G}(t)\right\}=P_{1}^{G T D G}(t) z^{5500}+P_{2}^{G T E G}(t) z^{4175}+P_{3}^{G T D G}(t) z^{2750}+P_{4}^{G T D G}(t) z^{1375}+P_{5}^{G T D G}(t) z^{0} .
$$

\section{The Main Switchboard}

As mentioned earlier, the main switchboard is a device with 2 states of performance: a fully operational state with a capacity $5500 \mathrm{KW}$ and a total failure corresponding to a capacity of 0 .

According to the Markov method and the state-transitions diagram (Fig. 3) we build the following system of differential equations:

$$
\left\{\begin{array}{l}
\frac{d p_{1}^{M S b}(t)}{d t}=-\lambda^{M S b} p_{1}^{M S b}(t)+\mu^{M S b} p_{2}^{M S b}(t), \\
\frac{d p_{2}^{M S b}(t)}{d t}=\lambda^{M S b} p_{1}^{M S b}(t)-\mu^{M S b} p_{2}^{M S b}(t) .
\end{array}\right.
$$

Initial conditions are: $p_{1}^{M S b}(0)=1 ; p_{2}^{M S b}(0)=0$.

A numerical solution for probabilities $p_{i}^{M S b}(t), i=1,2$ can be obtained for each of this system of

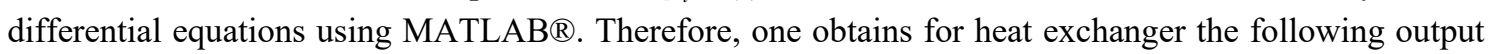
performance stochastic processes:

$$
\begin{aligned}
& \mathbf{g}^{M S b} \in\left\{g_{1}^{M S b}, g_{2}^{M S b}\right\}=\{5500,0\} ; \\
& \mathbf{p}^{M S b}(t)=\left\{p_{1}^{M S b}(t), p_{2}^{M S b}(t)\right\} .
\end{aligned}
$$

Having the set $\mathbf{g}^{M S b}, \mathbf{p}^{M S b}(t)$ one can define $L_{Z}$-transform, associated with the main switchboard's output performance stochastic process:

$$
L_{z}\left\{G^{M S b}(t)\right\}=p_{1}^{M S b}(t) z^{5500}+p_{2}^{M S b}(t) z^{0} .
$$

\section{Electric Energy Converter}

As mentioned earlier, the electric energy converter is a device with 2 states of performance: a fully operational state with a capacity $5500 \mathrm{KW}$ and a total failure corresponding to a capacity of 0 .

According to the Markov method and the state-transitions diagram (Fig. 3) we build the following system of differential equations:

$$
\left\{\begin{array}{l}
\frac{d p_{1}^{E E C}(t)}{d t}=-\lambda^{E E C} p_{1}^{E E C}(t)+\mu^{E E C} p_{2}^{E E C}(t) \\
\frac{d p_{2}^{E E C}(t)}{d t}=\lambda^{E E C} p_{1}^{E E C}(t)-\mu^{E E C} p_{2}^{E E C}(t)
\end{array}\right.
$$

Initial conditions are: $p_{1}^{E E C}(0)=1 ; p_{2}^{E E C}(0)=0$. 
A numerical solution for probabilities $p_{i}^{E E C}(t), i=1,2$ can be obtained for each of this system of differential equations using MATLAB $\AA$. Therefore, one obtains for heat exchanger the following output performance stochastic processes:

$$
\begin{aligned}
& \mathbf{g}^{E E C} \in\left\{g_{1}^{E E C}, g_{2}^{E E C}\right\}=\{5500,0\} ; \\
& \mathbf{p}^{E E C}(t)=\left\{p_{1}^{E E C}(t), p_{2}^{E E C}(t)\right\} .
\end{aligned}
$$

Having the set $\mathbf{g}^{E E C}, \mathbf{p}^{E E C}(t)$ one can define $L_{\mathrm{Z}}$-transform, associated with the electric energy converter's output performance stochastic process:

$$
L_{z}\left\{G^{E E C}(t)\right\}=p_{1}^{E E C}(t) z^{5500}+p_{2}^{E E C}(t) z^{0} .
$$

\section{E. Electric Motors' Subsystem}

As it was presented above, each electric motor half of capacity of the system. Each electric motor can be in one of two states: a fully operational state with a power of $2750 \mathrm{KW}$ and a state of total failure corresponding to a power of 0 .

According to the Markov method and state-transitions diagram (Fig. 3) we build the following system of differential equations for each pump $(i=1,2)$ :

$$
\left\{\begin{array}{l}
\frac{d p_{i 1}^{E M}(t)}{d t}=-\lambda^{E M} p_{i 1}^{E M}(t)+\mu^{E M} p_{i 2}^{E M}(t), \\
\frac{d p_{i 2}^{E M}(t)}{d t}=\lambda^{E M} p_{i 1}^{E M}(t)-\mu^{E M} p_{i 2}^{E M}(t) .
\end{array}\right.
$$

Initial conditions are: $p_{i 1}^{E M}(0)=1 ; p_{i 2}^{E M}(0)=0$.

A numerical solution for probabilities $p_{i 1}^{E M}(t)$ and $p_{i 2}^{E M}(t)(i=1,2)$ can be obtained for each of these 2 systems of differential equations using MATLAB®. Therefore, for each electric motor we obtain the following output performance stochastic processes:

$$
\left\{\begin{array}{l}
\mathbf{g}_{i}^{E M}=\left\{g_{i 1}^{E M}, g_{i 2}^{E M}\right\}=\{2750,0\} \\
\mathbf{p}_{i}^{E M}(t)=\left\{p_{i 1}^{E M}(t), p_{i 2}^{E M}(t)\right\}
\end{array}\right.
$$

Having the sets $\mathbf{g}_{i}^{E M}, \mathbf{p}_{i}^{E M}(t), i=1,2$ one can define for each electric motor $L_{\mathrm{Z}}$-transform, associated with the electric motor's output power performance stochastic process:

$$
\begin{gathered}
L_{z}\left\{G_{i}^{E M}(t)\right\}=p_{i 1}^{E M}(t) z^{g_{i 1}^{E M}}+p_{i 2}^{E M}(t) z^{g_{i 2}^{E M}} \\
=p_{i 1}^{E M}(t) z^{2750}+p_{i 2}^{E M}(t) z^{0} .
\end{gathered}
$$

Using the composition operator $\Omega_{\text {fpar }}$ for 2 electric motors, connected in parallel, one obtains the $L_{Z}$-transform $L_{z}\left\{G^{E M}(t)\right\}$ for the electric motor's sub-system, where the powers of $z$ are found as maximum values of powers of the corresponding terms:

$$
\begin{aligned}
L_{z}\left\{G^{E M}(t)\right\} & =\Omega_{f_{\text {par }}}\left(L_{z}\left\{g_{1}^{E M}(t)\right\}, L_{z}\left\{g_{2}^{E M}(t)\right\}\right) \\
& =\Omega_{f_{\text {par }}}\left(p_{11}^{E M}(t) z^{2750}+p_{12}^{E M}(t) z^{0}, p_{21}^{E M}(t) z^{2750}+p_{22}^{E M}(t) z^{0}\right) \\
& =p_{11}^{E M}(t) p_{21}^{E M}(t) z^{5500}+\left(p_{11}^{E M}(t) p_{22}^{E M}(t)+p_{21}^{E M}(t) p_{12}^{E M}(t)\right) z^{2750}+p_{12}^{E M}(t) p_{22}^{E M}(t) z^{0} .
\end{aligned}
$$


Using the following notations

$$
\begin{aligned}
& P_{1}^{E M}(t)=p_{11}^{E M}(t) p_{21}^{E M}(t) \\
& P_{2}^{E M}(t)=p_{11}^{E M}(t) p_{22}^{E M}(t)+p_{21}^{E M}(t) p_{12}^{E M}(t) \\
& P_{2}^{E M}(t)=p_{12}^{E M}(t) p_{22}^{E M}(t)
\end{aligned}
$$

one can obtain the resulting $L$ z-transform for the pump's sub-system in the following form

$$
L_{z}\left\{G^{E M}(t)\right\}=P_{1}^{E M}(t) z^{5500}+P_{2}^{E M}(t) z^{2750}+P_{3}^{E M}(t) z^{0} .
$$

\section{F. Multi-state model for Multi Power Source Traction Drive with Gas Turbine and Disel Generators}

All elements of the system are connected in series. Therefore, $L_{Z}$-transform, associated with the whole system is:

$$
\begin{aligned}
& L_{z}\left\{G^{M P D}(t)\right\}=\Omega_{f_{s e r}}\left(L_{z}\left\{G^{G T D G}(t)\right\}, L_{z}\left\{G^{M S b}(t)\right\}, L_{z}\left\{G^{E E C}(t)\right\}, L_{z}\left\{G^{E M}(t)\right\}\right) \\
&=\Omega_{f_{\text {ser }}}\left(P_{1}^{G T D G}(t) z^{5500}+P_{2}^{G T D G}(t) z^{4175}+P_{3}^{G T D G}(t) z^{2750}+P_{4}^{G T D G}(t) z^{1375}+P_{5}^{G T D G}(t) z^{0},\right. \\
&\left.p_{1}^{M S b}(t) z^{5500}+p_{2}^{M S b}(t) z^{0}, p_{1}^{E E C}(t) z^{5500}+p_{2}^{E E C}(t) z^{0}, P_{1}^{E M}(t) z^{5500}+P_{2}^{E M}(t) z^{2750}+P_{3}^{E M}(t) z^{0}\right)
\end{aligned}
$$

After simple algebra, where the powers of $z$ are found as minimum values of powers of corresponding terms, the final expression of the whole system's $L_{Z}$-transform is of the following form:

$$
L_{z}\left\{G^{M P D}(t)\right\}=P_{1}^{M P D}(t) z^{5500}+P_{2}^{M P D}(t) z^{4125}+P_{3}^{M P D}(t) z^{2750}+P_{4}^{M P D}(t) z^{2250}+P_{5}^{M P D}(t) z^{1375}+P_{6}^{M P D}(t) z^{0}
$$

where

$$
\begin{aligned}
& \left\{\begin{array}{l}
g_{1}^{M P D}=5500 K W \\
P_{1}^{M P D}(t)=P_{1}^{G T D G}(t) p_{1}^{M S b}(t) p_{1}^{E E C}(t) P_{1}^{E M}(t),
\end{array}\right. \\
& \left\{\begin{array}{l}
g_{2}^{\text {MPD }}=4125 K W, \\
P_{2}^{\text {MPD }}(t)=P_{2}^{G T D G}(t) p_{1}^{M S b}(t) p_{1}^{E E C}(t) P_{1}^{E M}(t) ;
\end{array}\right. \\
& \left\{\begin{array}{l}
g_{3}^{M P D}=2750 K W, \\
P_{3}^{M P D}(t)=P_{3}^{G T D G}(t) p_{1}^{M S b}(t) p_{1}^{E E C}(t) P_{1}^{E M}(t) ;
\end{array}\right. \\
& \begin{array}{l}
\left\{\begin{array}{l}
g_{4}^{M P D}=2250 K W, \\
P_{4}^{M P D}(t)=\left(P_{1}^{G T D G}(t)+P_{2}^{G T D G}(t)+P_{3}^{G}\right.
\end{array}\right. \\
\left\{\begin{array}{l}
g_{5}^{M P D}=1375 K W, \\
P_{5}^{M P D}(t)=P_{4}^{G T D G}(t) p_{1}^{M S b}(t) p_{1}^{E E C}(t) ;
\end{array}\right.
\end{array} \\
& \left\{\begin{array}{l}
g_{6}^{M P D}=0 K W, \\
P_{5}^{M P D}(t)=\left(1-P_{5}^{G T D G}(t)\right) p_{1}^{M S b}(t)\left(p_{1}^{E E C}(t) P_{3}^{E M}(t)+p_{1}^{E E C}(t)\right)+p_{2}^{M S b}(t)+P_{5}^{G T D G}(t) p_{1}^{M S b}(t) ;
\end{array}\right.
\end{aligned}
$$


Using the whole system's $L_{Z}$-transform one obtains MSS mean instantaneous availability for constant demand level $w$ in the following form:

- $\quad$ For $100 \%$ demand level $(w=5500 \mathrm{KW})$

$A_{w \geq 5500 K W}(t)=\sum_{g_{k} \geq 5500} P_{k}^{M P D}(t)=P_{1}^{M P D}(t)$

- $\quad$ For $50 \%$ demand level $(w=2750 \mathrm{KW})$

$A_{w \geq 2750 K W}(t)=\sum_{g_{k} \geq 2750} P_{k}^{M P D}(t)=\sum_{k=1}^{3} P_{k}^{M P D}(t)=P_{1}^{M P D}(t)+P_{2}^{M P D}(t)+P_{3}^{M P D}(t)$

G. Impuct of the Seasonal stochastic Demand on Multi-state model for Multi Power Source Traction Drive with Gas Turbine and Disel Generators

The system has to satisfy a seasonal stochastic demand that is described by two-state DSCT Markov process $W(t)$ with minimum performance level $w_{1}=2750 \mathrm{KW}$ and maximum performance level $w_{2}=5500$ KW. State-transition diagram for Markov process $W(t)$ is presented in Fig. 4.

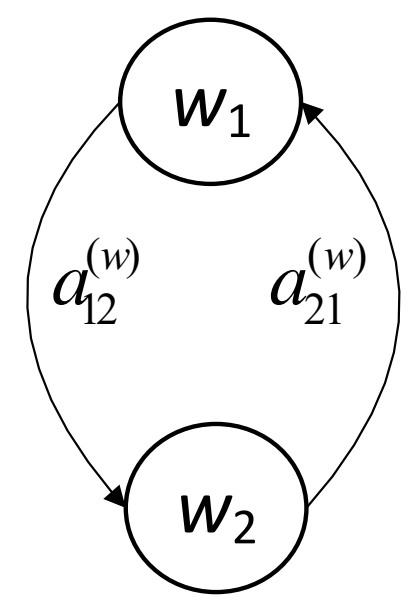

Figure 4. State-transition diagrams for demand Markov processes $W(t)$

Using this state-transitions diagram in accordance with the Markov method, we build the following systems of differential equations:

$$
\left\{\begin{array}{l}
\frac{d p_{w 1}(t)}{d t}=-a_{12}^{(w)} p_{1 g}(t)+a_{21}^{(w)} p_{2 w}(t), \\
\frac{d p_{w 2}(t)}{d t}=a_{12}^{(w)} p_{1 g}(t)-a_{21}^{(w)} p_{2 w}(t),
\end{array}\right.
$$

under initial conditions $\mathbf{p}_{w 0}=\left\{p_{w 1}(0), p_{w 2}(0)\right\}=\{1,0\}$.

A numerical solution for probabilities $p_{w 1}(t)$ and $p_{w 2}(t)$ can be obtained for this system of differential equations using MATLAB ${ }^{\circledR}$. Therefore, for seasonal stochastic demand we obtain the following output performance stochastic processes:

$$
\left\{\begin{array}{l}
\mathbf{g}_{W}=\left\{g_{w 1}, g_{w 2}\right\}=\{5500,2750\} \\
\mathbf{p}_{W}(t)=\left\{p_{w 1}(t), p_{w 2}(t)\right\}
\end{array}\right.
$$


Having the sets $\mathbf{g}_{W}, \mathbf{p}_{W}(t)$ one can define for seasonal stochastic demand $L_{Z}$-transform, associated with the stochastic process of seasonal stochastic demand:

$$
\begin{aligned}
L_{z}\left\{G_{W}(t)\right\} & =p_{w 1}(t) z^{g_{w 1}}+p_{w 2}(t) z^{g_{w 2}} \\
& =p_{w 1}(t) z^{5500}+p_{w 2}(t) z^{2750} .
\end{aligned}
$$

When the resulting stochastic process $Y(t)=f\{G(t), W(t)\}=G(t)-W(t)$ falls down to level zero such event is treated as a failure. Processes $G(t), W(t)$ are independent.

The problem is to find instantaneous availability and instantaneous mean performance deficiency for this aging multi-state production system under the seasonally changing stochastic demand.

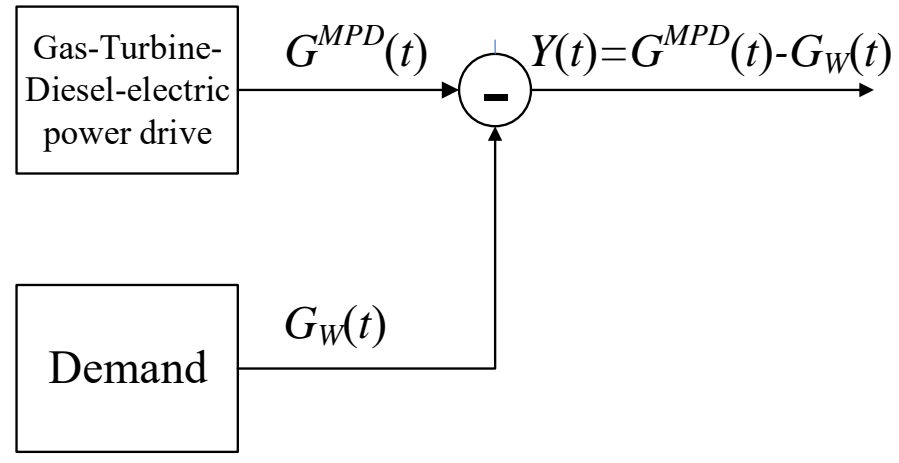

Figure 5. Block-diagram for the MSS reliability computation

In according to the $L_{Z}$-transform definition we obtain

$$
\begin{aligned}
& L_{z}\left\{G^{M P D}(t)\right\}=P_{1}^{M P D}(t) z^{5500}+P_{2}^{M P D}(t) z^{4125}+P_{3}^{M P D}(t) z^{2750}+P_{4}^{M P D}(t) z^{2250}+P_{5}^{M P D}(t) z^{1375}+P_{6}^{M P D}(t) z^{0} \\
& L_{z}\left\{G_{W}(t)\right\}=p_{w 1}(t) z^{5500}+p_{w 2}(t) z^{2750} .
\end{aligned}
$$

Now, we have

$$
\begin{aligned}
L_{z}\{Y(t)\}=L_{z}\left\{G^{M P D}(t)\right\}-L_{z}\left\{G_{W}(t)\right\}=\Omega_{f}\left\{L_{z}\left\{G^{M P D}(t)\right\}, L_{z}\left\{G_{W}(t)\right\}\right\} \\
=\Omega_{f}\left\{P_{1}^{M P D}(t) z^{5500}+P_{2}^{M P D}(t) z^{4125}+P_{3}^{M P D}(t) z^{2750}+P_{4}^{M P D}(t) z^{2250}+P_{5}^{M P D}(t) z^{1375}+P_{6}^{M P D}(t) z^{0} ; p_{w 1}(t) z^{5500}+p_{w 2}(t) z^{2750}\right\} \\
=\sum_{i=1}^{6} \sum_{j=1}^{2} P_{i}^{M P D}(t) p_{w j}(t) z^{\left(g_{i}-w_{j}\right)} \\
=P_{6}^{M P D}(t) p_{w 1}(t) z^{-5500}+P_{5}^{M P D}(t) p_{w 1}(t) z^{-4125}+P_{4}^{M P D}(t) p_{w 1}(t) z^{-3250}+\left(P_{3}^{M P D}(t) p_{w 1}(t) z^{-4125}+P_{6}^{M P D}(t) p_{w 2}(t)\right) z^{-2750} \\
\quad+\left(P_{2}^{M P D}(t) p_{w 1}(t)+P_{5}^{M P D}(t) p_{w 2}(t)\right) z^{-1375}+P_{4}^{M P D}(t) p_{w 2}(t) z^{-500}+\left(P_{1}^{M P D}(t) p_{w 1}(t)+P_{3}^{M P D}(t) p_{w 2}(t)\right) z^{0} \\
\quad+P_{2}^{M P D}(t) p_{w 2}(t) z^{1375}+P_{1}^{M P D}(t) p_{w 2}(t) z^{2750} .
\end{aligned}
$$

Based on the last expression we obtain MSS's instantaneous availability as probability of states with nonnegative powers:

$$
\begin{aligned}
A V(t) & =P_{1}^{M P D}(t) p_{w 1}(t)+P_{3}^{M P D}(t) p_{w 2}(t)+P_{2}^{M P D}(t) p_{w 2}(t)+P_{1}^{M P D}(t) p_{w 2}(t) \\
& =P_{1}^{M P D}(t)+\left(P_{2}^{M P D}(t)+P_{3}^{M P D}(t)\right) p_{w 2}(t)
\end{aligned}
$$

Note that in order to solve this problem by using straightforward Markov methods one should solve the system of 196 differential equations. 


\section{Power Performance Calculation}

Calculations were performed using the following failure and repair rates.

The failure rate and repair rate of the Gas Turbine engine are $\lambda^{G T E}=3.22$ year $^{-1} \mu^{G T E}=55$ year $^{-1}$ accordingly,

The failure rate and repair rate of the generator, connected to the Gas Turbine are $\lambda^{G T G}=0.004$ and $\mu^{G T G}=65$ year $^{-1}$.

The failure rate of each diesel engine is $\lambda^{D E}=4.99$ year $^{-1}$. The repair rate of the each diesel engine is $\mu^{D E}=180$ year $^{-1}$.

The failure rate of the generator is $\lambda^{G}=0.006$ year $^{-1}$. The repair rate of the generator is $\mu^{G}=50$ year $^{-1}$.

The failure rate of main switchboard is $\lambda^{M S b}=0.006$ year $^{-1}$. The repair rate of the each generator is $\mu^{M S b}=20$ year ${ }^{-1}$.

The failure rate of the electric energy converter is $\lambda^{E E C}=0.44$ year $^{-1}$. The repair rate of the electric energy converter is $\mu^{E E C}=25$ year $^{-1}$.

The failure rate of each electric motor is $\lambda^{E M}=0.0026$ year $^{-1}$. The repair rate of the each electric motor is $\mu^{E M}=100$ year $^{-1}$.

The transition intensities for the demand are as follows: $a_{12}^{(w)}=1095$ year-1, $a_{21}^{(w)}=487+200 \sin (2 \pi t)$ year ${ }^{-1}$.

As one can see the demand is seasonally changing - the maximum's duration is increasing at summer and decreasing at winter.

MSS mean instantaneous availability for different constant demand levels is presented in Figure 6.

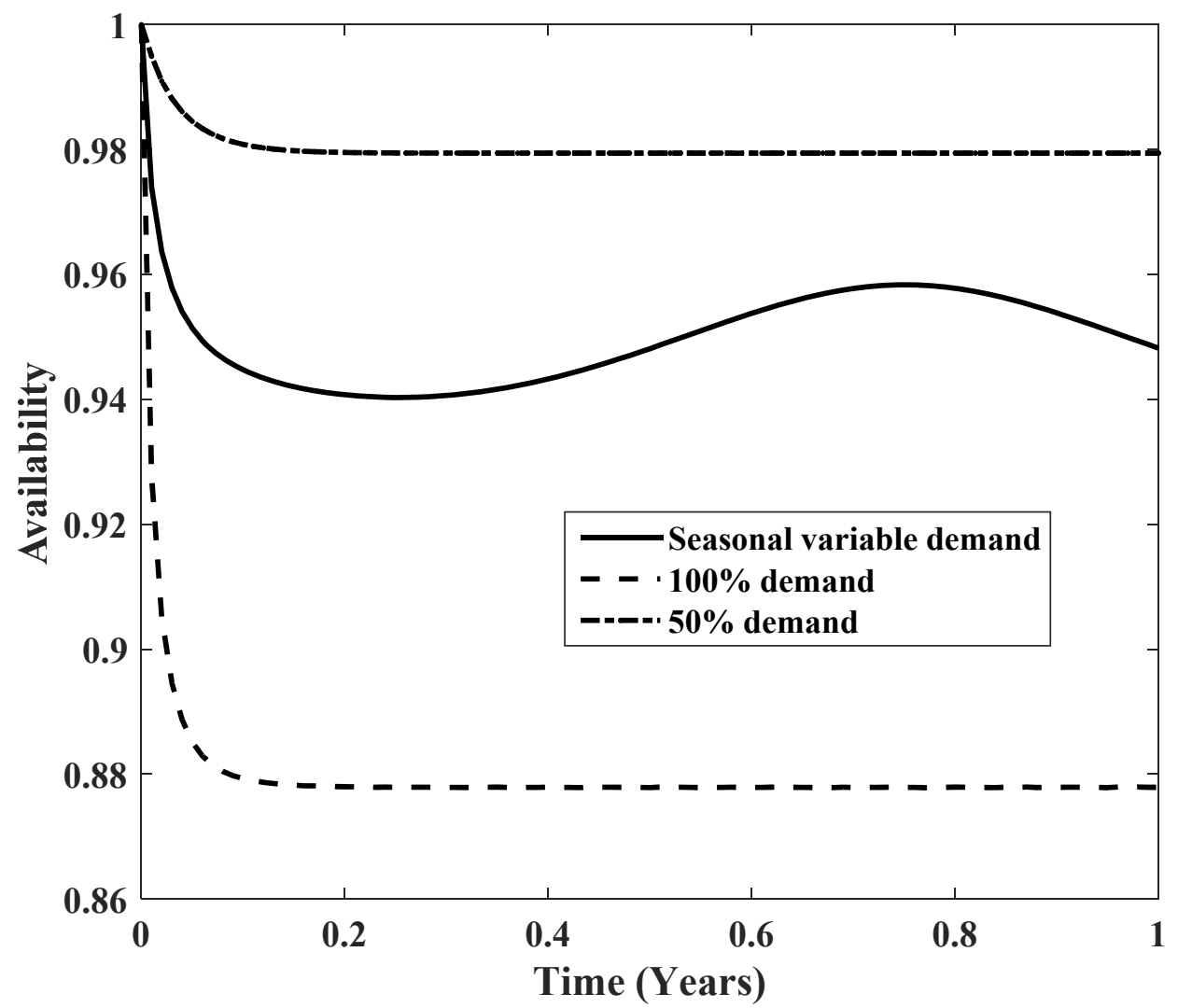

Figure 6. MSS mean instantaneous availability for different demand levels 
As one can see from Fig.5, the instantaneous availability depends on operation conditions and changes from $88 \%$ for $100 \%$ of required of generated power to $98.4 \%$ for $50 \%$ of required generated power. Availability curve for the seasonal demand depends on the ice and temperature conditions, presented in the Fig. 2.

\section{Conclusion}

In this paper, the $L_{Z}$-transform was applied to a real problem -availability assessment of a Multi Power Source Traction Drive using in variable seasonal conditions.

It was demonstrated, that the $L_{Z}$-transform method is well formalized and suitable for practical application in reliability engineering for a real-world MSSs analysis. It supports an engineering decisionmaking and determines a maintenance policy to provide a required power-performance level for complex multi-state Multi Power Source Traction Drive. The proposed method provides a drastic decrease in computational burden compared with a straightforward Markov method, which in this case study would have required to build and solve a model with 196 states.

The proposed approach allows an optimizing the number, type and arrangement of the power sources of traction drive, their characteristics and schemes of connection in terms of providing the maximum operational availability. More broadly, the proposed method can be used as a universal tool for evaluation and optimization of performance availability of electric propulsion systems in real operational conditions, considering all possibilities of its increasing, such as redundancy, monitoring, predictive control and type of maintenance strategy.

\section{References}

1. Bolvashenkov, I., Herzog, H.-G. (2016) Use of Stochastic Models for Operational Efficiency Analysis of Multi Power Source Traction Drives. In: Proceedings of the Second International Symposium on Stochastic Models in Reliability Engineering, Life Science and Operations Management (SMRLO'16), Ilia Frenkel and Anatoly Lisnianski (eds.), Beer Sheva, Israel, February 15-18, (2016) pp 124 - 130, IEEE CPS, 978-1-4673-9941-8/16, DOI 10.1109/SMRLO.2016.30

2. Bolvashenkov, I., Herzog, H.-G., Rubinraut, A., Romanovskiy, V. (2014) Possible Ways to Improve the Efficiency and Competitiveness of Modern Ships with Electric Propulsion Systems. In: (2014) IEEE Vehicle Power and Propulsion Conference (VPPC), Coimbra, Portugal, October 27-30, (2014), IEEE, pp. 1-9. DOI 10.1109/VPPC.2014.7007120

3. Lisnianski, A. (2012) $\mathrm{L}_{\mathrm{z}}$-transform for a Discrete-state Continuous-time Markov Process and its Application to Multi-state System Reliability. In: Recent Advances in System Reliability. Signatures, Multi-state Systems and Statistical Inference. A. Lisnianski and I. Frenkel, Eds. London: Springer, 2012, pp. 79-95.

4. Lisnianski, A., Frenkel, I. and Ding, Y. (2010) Multi-state System Reliability Analysis and Optimization for Engineers and Industrial Managers. London: Springer.

5. Ushakov, I. (1986) A Universal Generating Function. Soviet Journal of Computer and System Sciences, vol. 24 , pp. 37-49. 